T. Sasaki and O. Suzuki

Nagoya Math. J.

Vol. 67 (1977), 53-64

\title{
SOME APPLICATIONS OF SYNGE'S FORMULA TO THE THEORY OF SEVERAL COMPLEX VARIABLES
}

\author{
TAKESHI SASAKI AND OSAMU SUZUKI*
}

\section{§1. Introduction}

In [10] and [11], the second author proved the following theorem by using Synge's formula:

THEOREM I. Let $M$ be a kähler manifold with positive holomorphic bi-sectional curvature. Then every pseudoconvex domain in $M$ is a Stein manifold.

This seems, as far as we know, to be the first application of Synge's formula in the theory of several complex variables. As for Synge's formula, see $\S 2$.

In the present paper we shall give further applications of Synge's formula and show that essential parts of the well known theorems concerning curvature and pseudoconvexity can be proved systematically by using Synge's formula. First we prove the following theorem of R. E. Greene and $\mathrm{H}$. Wu [7] by using Theorem I and the result of J. Cheeger and D. Gromoll [2, Proposition 1.3. p. 416]:

THEOREM II. Let $M$ be a non-compact complete kähler manifold with positive sectional curvature. Then $M$ is a Stein manifold.

Secondly we shall be concerned with complete kähler manifolds with non-positive sectional curvature. We prove the following three theorems, the first of which is well known as the theorem of $\mathrm{H}$. Wu [13]:

THEOREM III. Let $M$ be a simply connected complete kähler manifold

Received May 11, 1976.

* The second author was a visiting member of the Research Institute for Mathematical Sciences, Kyoto University during a part of the period of the preparation of this work. 
with non-positive sectional curvature. Then $M$ is a Stein manifold.

THEOREM IV. Let $M$ be a simply connected complete kähler manifold with non-positive (resp. negative) sectional curvature. For a point $p_{0}$ we set $\phi(p)=d\left(p, p_{0}\right)$, where $d\left(p, p_{0}\right)$ denotes the distance between $p$ and $p_{0}$. Then $\phi(p)$ is a complete pseudoconvex (resp. s-pseudoconvex) function on $M-\left\{p_{0}\right\}$.

THEOREM V. Let $M$ be a complete kähler manifold with non-positive (resp. negative) sectional curvature. Then for any point $p$ there exist a neighborhood $U$ of $p$ and a system of pseudoconvex (resp. s-pseudoconvex) functions $\phi_{1}, \phi_{2}, \cdots, \phi_{r}$ on $U$ such that

$$
\phi(q)=\min _{j=1,2, \cdots, r} \phi_{j}(q) \quad \text { for } q \in U .
$$

At the end of $\S 3$, we give an example of a not simply connected complete kähler manifold with negative curvature which is not a Stein manifold.

In $\S 4$ we shall give examples of kähler manifolds which show difficulties of differential geometric characterization of Stein manifolds.

The authors would like to express their hearty thanks to Professors A. Morimoto and $\mathrm{K}$. Aomoto and Mr. S. Takeuchi for their valuable discussions during the preparation of the present paper.

\section{§2. Synge's formula and normal coordinates of kähler manifolds}

First we consider a Riemannian manifold $R$ of class $C^{\infty}$ with a metric $g$. Let $\mathscr{U}=\{U\}$ be a local coordinate covering of $R$ and $x^{1}, x^{2}, \cdots, x^{m}$ denote local coordinates on $U$. The connection induced from $g$ is denoted by

$$
\nabla_{\partial / \partial x^{i}} \frac{\partial}{\partial x^{j}}=\sum \Gamma_{i j}^{k} \frac{\partial}{\partial x^{k}}
$$

Let $P$ and $N$ be two submanifolds in $R$ and let $p$ and $q$ be two points on $P$ and $N$, respectively. Moreover, suppose that a geodesic $\sigma$ joining $p$ with $q$ is given, whose length is denoted by $\ell$. A $C^{\infty}$-mapping $\psi(\xi, t)$ from a rectangle $[-\varepsilon, \varepsilon] \times[0, \ell]$ to $R$ is called a variation of $\sigma$ if $\psi(\xi, t)$ defines a curve $C_{\xi}$ with $\psi(\xi, 0) \in P$ and $\psi(\xi, \ell) \in N$ for a fixed $\xi$ and especially, when $\xi=0, \psi(0, t)$ gives a parametrization of $\sigma$. By $\dot{\psi}(\xi, t)$ we denote the tangent vector of $C_{\xi}$ at $t$. Then the length of $C_{\xi}$ is given 
as follows :

$$
\ell(\xi)=\int_{0}^{\ell}\|\dot{\psi}(\xi, t)\| d t,
$$

where $\|\dot{\psi}(\xi, t)\|$ denotes the length of $\dot{\psi}(\xi, t)$. We define a vector field $X$ (resp. $Y$ ) along the map $\psi$ by

$$
\psi_{*}\left(D_{1}\right)=X \text { for } D_{1}=\frac{\partial}{\partial t} \quad \text { and } \quad \psi_{*}\left(D_{2}\right)=Y \text { for } D_{2}=\frac{\partial}{\partial \xi} .
$$

Letting $Y=\sum Y^{j} \frac{\partial}{\partial x^{j}} \circ \psi$ with $\left(\frac{\partial}{\partial x^{j}} \circ \psi\right)(\xi, t)=\left(\frac{\partial}{\partial x^{j}}\right)_{\psi(\xi, t)}$, we set

$$
\nabla_{D_{1}} Y=\sum \frac{\partial Y^{k}}{\partial t} \frac{\partial}{\partial x^{k}} \circ \psi+\sum \Gamma_{i j}^{k} \circ \psi \cdot Y^{j} \frac{\partial\left(x^{i} \circ \psi\right)}{\partial t} \frac{\partial}{\partial x^{k}} \circ \psi
$$

and

$$
\nabla_{D_{2}} Y=\sum \frac{\partial Y^{k}}{\partial \xi} \frac{\partial}{\partial x^{k}} \circ \psi+\sum \Gamma_{i j}^{k} \circ \psi \cdot Y^{j} \frac{\partial\left(x^{i} \circ \psi\right)}{\partial \xi} \frac{\partial}{\partial x^{k}} \circ \psi
$$

Then Synge's formula can be stated as follows:

LEMma 2.3. (The second variation of arc lengths). Suppose that $\|\dot{\psi}(0, t)\|=1$ for every $t$. Then we have

$$
\ell^{\prime \prime}(0)=\int_{0}^{\ell}\left\{g\left(\nabla_{D_{1}} \tilde{Y}, \nabla_{D_{1}} \tilde{Y}\right)-g(R(Y, X) X, Y)\right\}(0, t) d t+\left.g\left(\nabla_{D_{2}} Y, X\right)(0, t)\right|_{0} ^{\ell},
$$

where $\tilde{Y}=Y-g(Y, X) X$ and $R(Y, X)$ denotes the Riemannian curvature transformation.

For the proof of this Lemma, see [1, Corollary 1, p. 219].

Let $S$ be a submanifold in $R$. For each point $p \in S$, we define a bilinear symmetric mapping $\alpha_{p}: T_{p}(S) \times T_{p}(S) \rightarrow T_{p}(S)^{\perp}$ by

$$
\alpha_{p}\left(X_{0}, Y_{0}\right)=\text { the normal component of }\left(\nabla_{X} Y\right)_{p} \text {, }
$$

where $T_{p}(S), T_{p}(S)^{\perp}$ denote the tangent space of $S$ at $p$ and its orthogonal complement of $T_{p}(S)$ respectively and $X, Y$ are local vector fields in a neighborhood of $p$ such that $X_{p}=X_{0}$ and $Y_{p}=Y_{0} . \quad \alpha_{p}$ is called the second fundamental form of $S$ at $p$.

In the rest of this paper we confine ourselves to a study of kähler manifolds. Let $M$ be a kähler manifold of dimension $n$ with a kähler 
metric of class $C^{\infty}$

$$
d s^{2}=2 \sum g_{i j} d z^{i} \cdot d \bar{z}^{j} .
$$

The complex structure of $M$ is denoted by $J$.

The following proposition is well known (cf. [12]):

Proposition 2.4. For any point $p$ and for any geodesic starting at $p$, there exists a local coordinate neighborhood $U$ of $p$ and a system of local coordinates on $U$ with the following properties:

(1) $2 g_{i j}=\delta_{i j}+\sum K_{i j k l}(0) z^{k} \bar{z}^{l}+0\left(r^{3}\right)$ on $U$, where $r^{2}=\sum_{j=1}^{n}\left|z^{j}\right|^{2}$.

(2) Let $\psi$ be a parametrization of $\sigma$ by its arc length. Then $\dot{\psi}(0)=\frac{\partial}{\partial x^{1}}$ at $p$, where $x^{1}=\operatorname{Re} z^{1}$.

Proof. We sketch the outline of the proof given in [12]. Choose a system of local coordinates $w^{1}, w^{2}, \cdots, w^{n}$ at $p$. The metric tensor in terms of these coordinates is denoted by $\left\{g_{i j}\right\}$. Let $\left(\lambda_{k l}\right)$ be a matrix of numbers satisfying

$$
\sum_{i, j=1}^{n} g_{i j}(0) \lambda_{i k} \overline{\lambda_{j l}}=\delta_{k l}
$$

Define $u^{1}, u^{2}, \cdots, u^{n}$ by

$$
w^{i}=\sum_{k=1}^{n} \lambda_{i k} u^{k} \quad(i=1,2, \cdots, n) .
$$

Taking an arbitrary unitary matrix $\left(\alpha_{j}^{i}\right)$ and setting

$$
\begin{array}{r}
z^{i}=\sum_{j=1}^{n} \alpha_{j}^{i} u^{j}+\sum_{k=2}^{\infty} \sum_{i_{1}+i_{2}+\cdots+i_{n}=k} \alpha_{\imath_{1}, i_{2}, \cdots, i_{n}}^{i} u^{1_{1} 1} u^{2 i_{2}} \cdots u^{n^{i} n} \\
(i=1,2, \cdots, n),
\end{array}
$$

we determine $\left\{\alpha_{i_{1}, i_{2}, \cdots, i_{n}}^{i}\right\}$ so that the metric tensor with respect to these coordinates satisfies the condition (1) (see [12, Lemma 1]).

Now we take a geodesic $\sigma$ through $p$. By using a suitable unitary matrix $\left(\alpha_{k}^{i}\right)$, we can find a system of local coordinates $z^{1}, z^{2}, \cdots, z^{n}$ satisfying the conditions (1) and (2).

Letting $\Gamma_{i j}^{k}$ be connection coefficients with respect to real coordinates $x^{1}, y^{1}, \cdots, x^{n}, y^{n}$, where $z^{i}=x^{i}+\sqrt{-1} y^{i}(i=1,2, \cdots, n)$, we have the following

CoRollary 2.5. $\quad \Gamma_{j k}^{i}(0)=0(i, j, k=1,2, \cdots, 2 n)$. 


\section{§3. Proofs of Theorem II, III, IV and V}

Proof of Theorem II. By [2, Proposition 1.3] we have the following

Proposition 3.1. Let $R$ be a non-compact complete Riemannian manifold with non-negative sectional curvature. Then there exists a family of compact domains $\left\{C_{t}\right\}_{t \geq 0}$ such that

(1) $R=\cup_{t \geq 0} C_{t}$,

(2) for every $t C_{t}$ is totally convex,

(3) if $t_{1}<t_{2}$, then $C_{t_{1}} \subseteq C_{t_{2}}$ and $C_{t_{1}}=\left\{p \in C_{t_{2}}: d\left(p, \partial C_{t_{2}}\right) \geq t_{2}-t_{1}\right\}$ and $\partial C_{t_{1}}=\left\{p \in C_{t_{2}}: d\left(p, \partial C_{t_{2}}\right)=t_{2}-t_{1}\right\}$.

Here a domain $C_{t}$ is called totally convex if for any pair of two points $p$ and $q$ in $C_{t}$ and for any geodesic $\sigma$ joining $p$ with $q, \sigma$ is contained completely in $C_{t}$.

Now we consider our complete kähler manifold $M$ with positive sectional curvature. Then for a family of domains $\left\{C_{t}\right\}$ satisfying the conditions in Proposition 3.1, we can prove the following

Proposition 3.2. $C_{t}$ is a pseudoconvex domain for every $t$.

Proof. Let $t^{\prime}$ be a positive constant with $t^{\prime}>t$. Let $\psi(p)=-d\left(p, \partial C_{t^{\prime}}\right)$ for $p \in C_{t^{\prime}}$. Then by using (2) in Proposition 3.1 and Theorem 1.10 in [2], for any geodesic $\sigma(s)$ in $C_{t}$, which is parametrized by its arc length $s$, we have

$$
\psi \circ \sigma\left(\alpha s_{1}+\beta s_{2}\right) \leq \alpha \psi \circ \sigma\left(s_{1}\right)+\beta \psi \circ \sigma\left(s_{2}\right),
$$

where $\alpha \geq 0, \beta \geq 0$ and $\alpha+\beta=1$. This implies that $\psi$ is a geodesically convex function on $C_{t}$, in the sense of [7, p. 641]. Then by Theorem 3 in $[7$, p. 652], we see that $\psi$ is a pseudoconvex function in the interior of $C_{t^{\prime}}$. By (3) in Proposition 3.1, we find that

$$
C_{t}=\left\{\psi \leq t-t^{\prime}\right\}
$$

which proves the assertion.

Then by Theorem I, we see that $C_{t}$ is a Stein manifold. So $M$ is also a Stein manifold.

Remark 1. From the manner given in the proof, we can also prove that if $M$ is a complete kählar manifold with positive holomorphic bisectional curvature and nonnegative sectional curvature, then $M$ is a 
Stein manifold.

Proof of Theorem III. Let $T_{p}(M)$ be the tangent space at $p$. Suppose a submanifold $S$ is given. For a point $p \in S$, we set

$$
H_{p}(S)=\left\{X_{0} \in T_{p}(S): J X_{0} \in T_{p}(S)\right\}
$$

Fix a point $p_{0}$ in $M$ and consider the exponential mapping exp: $T_{p_{0}} M$ $\rightarrow M$. Then by the theorem of J. Hadamard and E. Cartan, exp gives a diffeomorphism for every $p_{0}$. So we see that

$$
M \cong R^{2 n}
$$

Set $\phi(p)=d\left(p, p_{0}\right)$. Then we see that $\phi(p)$ is a function of $C^{\infty}$-class on $M-\left\{p_{0}\right\}$. Now we prove that $M_{c}=\{\phi<c\}$ is a Stein manifold for every c. For this we show that $S=\partial M_{c}$ satisfies the condition of Levi-Krzoska at every point in $S$. First we prove the following

Proposition 3.4. For any point $p \in S$ and for any $Y_{0} \in T_{p}(S)$, we have

$$
g\left(\alpha\left(Y_{0}, Y_{0}\right), X_{0}\right)<0,
$$

where $X_{0}$ denotes the outer normal vector of $S$ at $p$.

Proof. Let $p \in S$. Because $M$ is simply connected, there exists a unique geodesic $\sigma$ joining $p_{0}$ with $p . \quad \sigma$ is assumed to be parametrized by its arc length. Take $Y_{0} \in T_{p}(S)$. Then there exists a variation $\psi$ of $\sigma$ with the properties: (1) $\psi(\xi, 0)=p_{0}$ and $\psi(\xi, c) \in S$, (2) $X(0, t)$ is the tangent vector of $\sigma$, (3) $Y(0, c)=Y_{0}$ and (4) $\tilde{Y}=Y$, where $X, Y$ and $\tilde{Y}$ are defined as in $\S 2$. For the existence of such a variation, see [1, p. 217, Remark]. Then by Lemma 2.3 we have

$$
\ell^{\prime \prime}(0)=\int_{0}^{c}\left\{g\left(\nabla_{D_{1}} Y, \nabla_{D_{1}} Y\right)-g(R(Y, X) X, Y)\right\}(0, t) d t+\left.g\left(\nabla_{D_{2}} Y, X\right)(0, t)\right|_{0} ^{c} .
$$

By construction, $g\left(\nabla_{D_{2}} Y, X\right)(0,0)=0$. On the other hand, since $\ell(\xi)$ is maximal at $\xi=0, \ell^{\prime \prime}(0) \leq 0$. Hence in view of $g\left(\nabla_{D_{2}} Y, X\right)(0, c)=g\left(\nabla_{Y_{0}} Y_{0}, X_{0}\right)$ and $X(0, c)=X_{0}$, we have

$$
g\left(\nabla_{Y_{0}} Y_{0}, X_{0}\right) \leq-\int_{0}^{c} g\left(\nabla_{D_{1}} Y, \nabla_{D_{1}} Y\right)(0, t) d t .
$$

Since we easily find that $\nabla_{D_{1}} Y \neq 0$, we complete the proof of Proposition 


\section{4.}

By R. Hermann [9], we see that

$$
4 \partial \bar{\partial} \phi\left(W_{0}, \bar{W}_{0}\right)=-\left\{g\left(\alpha\left(Y_{0}, Y_{0}\right), X_{0}\right)+g\left(\alpha\left(J Y_{0}, J Y_{0}\right), X_{0}\right)\right\}
$$

for any tangent vector $W_{0}$ to $S$ of type (1.0), where $Y_{0}=W_{0}+\bar{W}_{0} \in H_{p}(S)$. This shows that $M_{c}$ is a $s$-pseudoconvex domain in $M$. To complete the proof of Theorem III, it is sufficient to prove the following

Proposition 3.5. For every $c, M_{c}$ admits no exceptional analytic sets in the sense of H. Grauert [4].

Proof. Suppose that $M_{c}$ admits an exceptional set $E$. Then there exists a constant $c$ such that $E \subset M_{c}$ and $E \not \subset M_{c^{\prime}}$ for $c^{\prime}<c$. Choose a point $p \in E \cap \partial M_{c}$. Then there exists a neighborhood $U$ of $p$ and a $s$ pseudoconvex function $u$ on $U$ such that $U \cap M_{c}=\{u<0\}$. Restricting $u$ to $E \cap U$, we obtain a s-pseudoconvex function $u^{\prime}$ on $E \cap U$. Then by the maximum principle, we see that $u^{\prime}=0$. Hence $E \subset \partial M_{c}$ holds in $U$, which contradicts the Levi-Krzoska condition at $p$.

Proof of Theorem IV. We consider $\phi(p)$ as in the proof of Theorem III. Let $p \in M$ and let $\sigma$ be a unique geodesic between $p_{0}$ and $p$. We choose a local coordinate system $z^{1}, z^{2}, \cdots, z^{n}$ at $p$ satisfying the properties $(1) \sim(3)$ in Proposition 2.4. Take $Y_{0} \in T_{p}(M)$ and set $Z_{0}=J Y_{0}$. Let $L$ be a complex line which is spanned by $Y_{0}$ and $Z_{0}$, which is parametrized as follows:

$$
z^{1}=a_{1} \lambda, z^{2}=a_{2} \lambda, \cdots, z^{n}=a_{n} \lambda,
$$

where $\left|a_{1}\right|^{2}+\left|a_{2}\right|^{2}+\cdots+\left|a_{n}\right|^{2}=1$.

Take a point $r \in L$ with a parameter $\lambda=\xi+\sqrt{-1} \eta$. Then there exists a unique geodesic joining $p_{0}$ with $r$ which attains its distance. We denote it by $\psi(\xi, \eta, t)$. By this we can construct a $C^{\infty}$-class variation $\psi(\xi, \eta, t)$ of $\sigma$ such that $\psi_{*}\left(\frac{\partial}{\partial \xi}\right)(0,0, c)=Y_{0}$ and $\psi_{*}\left(\frac{\partial}{\partial \eta}\right)(0,0, c)=Z_{0}$. We set $\psi_{*}\left(D_{1}\right)=X$ for $D_{1}=\frac{\partial}{\partial t}$ and $\psi_{*}\left(D_{2}\right)=Y$ for $D_{2}=\frac{\partial}{\partial \xi}$ and $\psi_{*}\left(D_{3}\right)=Z$ for $D_{3}=\frac{\partial}{\partial \eta}$.

Now set 


$$
\phi(\xi, \eta)=\int_{0}^{c}\|\dot{\psi}(\xi, \eta, t)\| d t
$$

Then, by Lemma 2.3 we obtain

$$
\begin{aligned}
\frac{\partial^{2} \phi}{\partial \xi^{2}}(0,0)= & \int_{0}^{c}\left\{g\left(\nabla_{D_{1}} \tilde{Y}, \nabla_{D_{1}} \tilde{Y}\right)-g(R(Y, X) X, Y)\right\}(0,0, t) d t \\
& +\left.g\left(\nabla_{D_{2}} Y, X\right)(0,0, t)\right|_{0} ^{c} .
\end{aligned}
$$

First we compute the last term. In view of $p_{0}=\psi(\xi, \eta, 0),\left(\nabla_{D_{2}} Y\right)(0,0,0)$ $=0$ (see, (2.2)). Thus we have $g\left(\nabla_{D_{2}} Y, X\right)(0,0,0)=0$. By (2) in Proposition 2.4,

$$
\frac{d}{d t} \psi(0,0, c)=\frac{\partial}{\partial x^{1}}(p)
$$

Moreover, by (1) in Proposition 2.4 and Corollary 2.5,

$$
g\left(\frac{\partial}{\partial x^{i}}, \frac{\partial}{\partial x^{j}}\right)(p)=\delta_{i j}, \quad g\left(\frac{\partial}{\partial x^{i}}, \frac{\partial}{\partial y^{j}}\right)(p)=0 \quad \text { and } \quad \Gamma_{j k}^{i}(p)=0 .
$$

Thus we see that $g\left(\nabla_{D_{2}} Y, X\right)(0,0, c)=\frac{\partial^{2}\left(x^{1} \circ \psi(\xi, 0, c)\right)}{\partial \xi^{2}}$ by (2.2). Then by using $x^{1} \circ \psi(\xi, 0, c)=\operatorname{Re} \alpha_{1} \xi$, we see that

$$
\left.g\left(\nabla_{D_{2}} Y, X\right)(0,0, t)\right|_{0} ^{c}=0 .
$$

Hence we obtain

$$
\frac{\partial^{2} \phi}{\partial \xi^{2}}(0,0)=\int_{0}^{c}\left\{g\left(\nabla_{D_{1}} \tilde{Y}, \nabla_{D_{1}} \tilde{Y}\right)-g(R(Y, X) X, Y)\right\}(0,0, t) d t
$$

So if the sectional curvature is non-positive (resp. negative), this is nonnegative (resp. positive). In the same manner we have $\frac{\partial^{2} \phi}{\partial \eta^{2}}(0,0) \geq 0$ (resp. $>0)$. Therefore

$$
\Delta(p)=\left(\frac{\partial^{2} \phi}{\partial \xi^{2}}+\frac{\partial^{2} \phi}{\partial \eta^{2}}\right)(0,0) \geq 0 \quad(\text { resp. }>0) .
$$

This proves the assertion.

Remark 2. We can give another proof of Theorem III as follows: We consider $M_{c}$ and $S$ as in the proof of Theorem III. Take $Y_{0} \in H_{p}(S)$ and consider a complex line $L$ as in the proof of Theorem IV. Then 
we have $\left(\nabla_{D_{1}} Y\right)(0,0, c) \neq 0$. So by $(3.7) \frac{\partial^{2} \phi}{\partial \xi^{2}}(0,0)>0$. Also we have $\frac{\partial^{2} \phi}{\partial \eta^{2}}(0,0)>0$. Thus $\Delta(p)>0$, which implies that $M_{c}$ satisfies the condition of Levi-Krzoska.

Remark 3. By (3.7) we obtain

$$
\Delta \phi(p) \geq-\int_{0}^{c}\{g(R(Y, X) X, Y)+g(R(Z, X) X, Z)\}(0,0, t) d t .
$$

Unfortunately we do not know that $Z=J Y$. Therefore we cannot assert a similar result to Theorem III under the assumption of non-positive holomorphic bisectional curvature. Also see $\S 4$.

Proof of Theorem V. Fix a point $p_{0}$ and consider $\phi(p)=d\left(p, p_{0}\right)$. For any point $p$, there exists a finite number of geodesics $\left\{\sigma_{i}\right\}$ which attain the distance between $p_{0}$ and $p$. Also for each pair $i$ and $j(i \neq j)$ the homotopy class of $\sigma_{i}^{-1} \circ \sigma_{1}$ is different from the one of $\sigma_{j}^{-1} \circ \sigma_{1}$. These follow from the fact that any negatively curved Riemannian manifold admits no conjugate points (see [1]). Consider $\sigma_{i}$. Let $z^{1}, z^{2}, \cdots, z^{n}$ be a system of local coordinates at $p$ which is chosen for $\sigma_{i}$ in Proposition 2.4. Let $L$ be a complex line through $p$ which is parametrized as in (3.6). Now we construct a variation $\psi_{i}$ of $\sigma_{i}$ as in the proof of Theorem IV. The length of the curve $\sigma_{i}$ is denoted by $\phi_{i}(\xi, \eta)$. Then we obtain

$$
\left(\frac{\partial^{2} \phi_{i}}{\partial \xi^{2}}+\frac{\partial^{2} \phi_{i}}{\partial \eta^{2}}\right)(0,0) \geq-\int_{0}^{c}\{g(R(Y, X) X, Y)+g(R(Z, X) X, Z)\}(0,0, t) d t,
$$

$X, Y, Z$ denoting the vector fields along a curve $\sigma_{i}$. It is easily shown that on a small neighborhood of $p, \phi(p)$ can be expressed as

$$
\phi(p)=\min _{i=1,2, \cdots, r} \phi_{i}
$$

This proves Theorem $\mathrm{V}$.

Remark 4. $\phi(p)$ is not always a function of $C^{\infty}$-class. Moreover, $\phi(p)$ is not always a pseudoconvex function. For example, if $M$ is a compact kähler manifold with non-positive sectional curvature, then $\phi(p)$ can never be a pseudoconvex function.

Remark 5. A non-compact complete kähler manifold with negative 
sectional curvature is not always a Stein manifold. We give the following example communicated to the authors by $\mathrm{K}$. Aomoto.

ExAmple. Let $D=\left\{\left(z_{1}, z_{2}\right) \in C^{2}:\left|z_{1}\right|^{2}+\left|z_{2}\right|^{2}<1\right\}$ and let $\Gamma$ be an arithmetic discontinuous group on $D$ such that $D / \Gamma$ is a non-compact complex manifold. Then the Bergmann metric on $D$ induces a complete kähler metric with negative sectional curvature on $M=D / \Gamma$. On the other hand, it is well known that for some $\Gamma, M$ has a compactification $\bar{M}$ such that $\bar{M}$ is a compact analytic space and $\bar{M}-M$ is a finite number of points. So $M$ can never be a Stein manifold.

\section{§4. Some examples of kähler manifolds concerning differential geometric char- acterization of Stein manifolds}

By giving examples of kähler manifolds, we shall show that differential geometric characterization of Stein manifolds is not easy.

First, we give an example which shows that a Stein manifold does not always admit a complete kähler metric of non-positive (resp. positive) curvature. For this we remark on the following: Let $M$ be a noncompact simply connected kähler manifold with non-positive (resp. positive) curvature. Then by (3.3) (resp. R. Gromoll and W. Meyer [8]), we see that

$$
H^{i}(M, Z)=0 \quad \text { for } i=1,2, \cdots, 2 n .
$$

Example 1 (S. Takeuchi). Let $G$ be a subgroup of $S L(2, C)$ defined by

$$
G=\left\{\left(\begin{array}{cc}
z & 0 \\
0 & z^{-1}
\end{array}\right): z \in C^{*}\right\}
$$

Then by the theorem of M. Rosenlicht and C. Chevalley, we see that $M=S L(2, C) / G$ is an affine algebraic manifold. So $M$ is a Stein manifold. The homotopy type of $M$ is identical with the one of the real 2dimensional sphere. Hence $M$ is simply connected and $H^{2}(M, Z)=Z$. Therefore by (4.1) $M$ does not admit any complete kähler metric with non-positive (resp. positive) curvature.

In view of the example given at the end of $\S 3$, we see that the condition of non-positive curvature is not sufficient condition for a kähler manifold to be Stein if it is not simply connected.

As for a necessary condition, we know the following proposition which 
is due to S. I. Goldberg and S. Kobayashi [3]:

Proposition 4.2. Let $M$ be a Stein manifold. Then $M$ admits a complete kähler metric with non-positive holomorphic bisectional curvature.

But the condition of non-positivity of holomorphic bisectional curvature is not a sufficient condition for a not simply connected kählar manifold to be Stein. In fact, we have the following

EXAMPLE 2 (H. Grauert [5]). Let $C$ be a compact non-singular algebraic curve of genus $g(g \geq 2)$ and let $F$ be a topologically trivial holomorphic line bundle on $C$. With a fine covering $\left\{V_{\lambda}\right\}$ of $C, F$ may be expressed as

$$
F=\left\{f_{\lambda \mu}\right\}, \text { where }\left|f_{\lambda \mu}\right|=1 \text {. }
$$

The fibre coordinate on $V_{\lambda}$ is denoted by $\zeta_{\lambda}$. Set

$$
h=\left|\zeta_{\varkappa}\right|^{2} \text { and } V_{\varepsilon}=\{h<\varepsilon\} \text {. }
$$

$h$ is a $C^{\infty}$-function on $F$ and $V_{s}$ is a system of tubular neighborhoods of the zero section. We denote by $\mathcal{O}(M)$ the algebra of holomorphic functions on a complex manifold $M$ and by $D$ the unit disc. Then, by H. Grauert [5] we have the following

Proposition 4.4. (I) If $F^{k}$ is analytically trivial for some $k$ $(k \neq 0)$, then $\mathcal{O}\left(V_{s}\right)=\mathcal{O}(D)$, where $F^{k}$ is the $k$-th tensor product of $F$. (I) If $F^{k}$ is not analytically trivial for any $k(k \neq 0)$, then $\mathcal{O}(V)=C$.

Now we show that $V_{\varepsilon}$ admits a complete kähler metric $d s^{2}$ with nonpositive sectional curvature. In view of the definition of holomorphic bisectional curvature, this means that $V_{c}$ admits a complete kähler metric with non-positive holomorphic bisectional curvature. Let $\underline{d s}^{2}$ be the Poincaré distance on $C$. By

$$
d s^{2}=\pi^{*}\left(\underline{d s^{2}}\right)+(1-h / \varepsilon)^{-2} d \zeta_{\lambda} d \bar{\zeta}_{\lambda}
$$

gives a desired metric, where $\pi$ denotes the natural projection $\pi: F \rightarrow C$.

We finish our study with the following problem:

Problem. Is a simply connected kähler manifold $M$ a Stein manifold if and only if $M$ admits a complete kähler metric with non-positive holomorphic bisectional curvature? 


\section{REFERENCES}

[1] Bishop, R. L. and Crittenden, R. J., Geometry of Manifolds, Academic Press, New York, 1964.

[2] Cheeger, R. I. and Gromoll, R., On the structure of complete manifolds of nonnegative curvature, Ann. of Math. 96 (1974), 414-443.

[ 3 ] Goldberg, S. I. and Kobayashi, S.: On holomorphic bisectional curvature, J. Diff. Geometry 1 (1967), 225-233.

[ 4 ] Grauert, H., Über Modifikationen und die exzeptionelle analytische Mengen, Math. Ann. 146 (1962), 331-368.

[5] — , Bemerkenswerte pseudokonvexe Mannigfaltigkeiten, Math. Zeit. 81 (1964), $377-391$.

[6] Greene, R. E. and Wu, H., On the subharmonicity and plurisubharmonicity of geodesically convex functions, Indiana Univ. Math. J. 22 (1973), 641-653.

[7] - Approximation theorems, $C^{\infty}$-convex exhaustions and manifolds of positive curvature, Bull. Amer. Math. Soc. 81 (1975), 101-104.

[ 8 ] Gromoll, R. and Meyer, W., On complete open manifolds of positive curvature, Ann. of Math. 90 (1969), 75-90.

[9] Hermann, R., Convexity and pseudoconvexity for complex manifolds, J. Math. Mech. 13 (1964), 667-672.

[10] Suzuki, O., Pseudoconvex domains on a kähler manifold with positive holomorphic bisectional curvature, to appear in Publ. Res. Inst. Math. Sci., 12.

[11] Suzuki, O., Supplement to "Pseudoconvex domains on a kähler manifold with positive holomorphic bisectional curvature", to appear in Publ. Res. Inst. Math. Sci., 12.

[12] Takeuchi, A., Domains pseudoconvexes sur les variétés kähleriennes, J. Math. Kyoto Univ. 6-3 (1967), 323-357.

[13] Wu, H., Negatively curved kähler manifolds, Notices Amer. Math. Soc. 14 (1967), 515.

Nagoya University
and
Nihon University 